\title{
O USO DE PSEUDOPOTENCIAIS E MODELOS HF/MP2/DFT NA PREVISÃO DE FREQÜÊNCIAS VIBRACIONAIS EM COMPLEXOS METÁLICOS
}

\author{
Tiago Giannerini, Claudio Alberto Téllez Soto e Eduardo Hollauer* \\ Instituto de Química, Universidade Federal Fluminense, Outeiro de São João Batista, s/n, 24020-150 Niterói - RJ
}

Recebido em 20/2/03; aceito em 1/9/03

\begin{abstract}
THE USE OF PSEUDOPOTENTIALS AND HF/MP2/DFT MODELS FOR THE PREDICTION OF VIBRATIONAL FREQUENCIES OF METAL COMPLEXES. Four different pseudopotentials and three methodologies were employed in the calculation of the geometry and the frequencies of metal complexes like $\left[\mathrm{M}\left(\mathrm{NH}_{3}\right)_{2} \mathrm{X} /[\mathrm{X}=\right.$ halogen, $\mathrm{M}=\mathrm{Zn}, \mathrm{Cd}], \operatorname{and}\left[\mathrm{Hg}\left(\mathrm{NH}_{3}\right)_{2}\right] \mathrm{Cl}_{2}$. The vibrational assignments were carefully checked and compared to the theoretically calculated ones. Graphical procedures were employed to estimate family errors and their average behavior. The calculated results show the SBK-X basis set with the best results for the geometries and calculated frequencies, for individual species and statistical results. Its use is recommend, mainly if the neighborhood atoms are described with similar pseudopotentials. Excellent results were also obtained with the Hay and Wadt pseudopotential.
\end{abstract}

Keywords: Ab initio calculation; vibrational frequencies; metal complexes.

\section{INTRODUÇÃO}

O desenvolvimento computacional e metodológico das últimas décadas trouxe grande impulso à área da espectroscopia vibracional, seja na etapa de aquisição de dados em laboratório, seja na interpretação de seus resultados. Um de seus usos mais freqüentes é no cálculo teórico de freqüências de vibração ${ }^{1}$ que, conjuntamente à análise de coordenadas normais (ACN), são empregadas na atribuição e interpretação de espectros nesta região.

Recentemente, o procedimento de realizar a atribuição espectroscópica na região do infravermelho ganhou força com inúmeros estudos sistemáticos visando calibrar métodos ab initio para este fim. O emprego de bases estendidas, o tratamento da energia de correlação com métodos pós-SCF, como a teoria de perturbações MollerPlessett (MP2), a interações de configuração (CI) e o emprego do funcional densidade (DFT), estão entre as novas iniciativas. Na literatura encontramos amplas revisões do assunto para o modelo $a b$ initio $^{2-5}$ que, via de regra, exibe erros não superiores a $10 \%$.

Em outra abordagem, trabalhos envolvendo métodos baratos, como semi-empíricos acoplados ao tratamento de erros médios, mostraram grande sucesso na identificação de tendências de família e atribuições duvidosas ${ }^{6-10}$. Dewar analisou o sucesso do método MINDO/3 na previsão de freqüências vibracionais de compostos $\operatorname{orgânicos}^{6}$. Healy e Holder ${ }^{7}$ analisaram as frequiências AM1 de 42 moléculas orgânicas, enquanto Coolidge et al. ${ }^{8}$ compararam o comportamento dos modelos AM1, PM3, MNDO e MINDO/3 na previsão de frequiências em 61 compostos orgânicos e inorgânicos. Fausto 9 analisou o desempenho dos modelos AM1, PM3 e MNDO no cálculo de frequiências em compostos contendo o elemento enxofre. Em trabalho anterior realizado por Ramos et $a l .{ }^{10}$, verificamos, em uma das mais completas avaliações da literatura, que um tratamento de erros médios para as frequiências no modelo AM1 era capaz de reduzir à metade, em muitos casos, o erro de cálculo. Neste caso, é possível obter erros médios em torno de $5 \%$.
Em que pese os excelentes resultados obtidos para compostos orgânicos, não se obteve igual sucesso com compostos organo-metálicos, complexos inorgânicos e clusters. A razão desta dificuldade tem origem em alguns problemas de cálculo, o maior tamanho molecular, a proximidade energética dos orbitais e a necessidade de um amplo conjunto de base, sem o que não se obtém resultados confiáveis. Estas condições acarretam um custo computacional que é uma ordem de grandeza superior àquela de compostos orgânicos com igual número de átomos, com o agravante de crescente complexidade de interpretação trazida pelo elevado número de alternativas metodológicas, associadas ao tratamento desta propriedade.

Recentemente, Téllez e seu grupo conduziram um detalhado estudo do espectro vibracional de complexos metálicos de amônia e halogeneos ${ }^{11-18}$. Diversos complexos foram sintetizados, dentre eles o diamindiclorozinco(II) $\left[\mathrm{Zn}\left(\mathrm{NH}_{3}\right)_{2} \mathrm{Cl}_{2}\right]^{11}$, diamindibromozinco(II) $\left[\mathrm{Zn}\left(\mathrm{NH}_{3}\right)_{2} \mathrm{Br}_{2}\right]^{12}$, diamindiiodozinco(II) $\left[\mathrm{Zn}\left(\mathrm{NH}_{3}\right)_{2} \mathrm{I}_{2}\right]^{13}$, diamindiclorocádmio(II) $\left[\mathrm{Cd}\left(\mathrm{NH}_{3}\right)_{2} \mathrm{Cl}_{2}\right]^{14-15}$, diamindibromocádmio(II) $\left[\mathrm{Cd}\left(\mathrm{NH}_{3}\right)_{2} \mathrm{Br}_{2}\right]^{16}$, diamindiiodocádmio(II) $\left[\mathrm{Cd}\left(\mathrm{NH}_{3}\right)_{2} \mathrm{I}_{2}\right]^{17}$ e o cloreto de diaminmercúrio(II) $\left[\mathrm{Hg}\left(\mathrm{NH}_{3}\right)_{2}\right] \mathrm{Cl}_{2}{ }^{18}$, dentre outros. Estes complexos foram sintetizados, caracterizados por difração de raios-X, e detalhadamente estudados por espectroscopia de absorção na região do infravermelho e de espalhamento Raman. Nestes estudos foram realizados experimentos de substituição isotópica de hidrogênio/nitrogênio, cujos resultados foram submetidos à análise de coordenadas normais. Apesar do minucioso estudo, ainda existem dúvidas no tocante a atribuição das bandas de mais baixa energia e aos modos de vibração de esqueleto. Deve-se destacar que dentre os membros desta família há compostos de estrutura planar, aqueles com os grupos amino em cadeia linear $\left(\mathrm{Cd}\left(\mathrm{NH}_{3}\right)_{2} \mathrm{X}_{2}, \mathrm{e}\left[\mathrm{Hg}\left(\mathrm{NH}_{3}\right)_{2}\right]^{2+}\right)$, enquanto outros são tetraédricos $\left(\mathrm{Zn}\left(\mathrm{NH}_{3}\right)_{2} \mathrm{X}_{2}\right)$, o que oferece um rico conjunto de possibilidades para avaliação do comportamento dos métodos de cálculo ab initio empregando pseudopotenciais. Adicionalmente, deve-se observar a inexistência de cálculos realizados para esta família comparando os diversos métodos teóricos disponíveis.

Recentemente Lin Zhang et al. estudaram diversos complexos amin-metálicos do tipo $\mathrm{M}\left(\mathrm{NH}_{3}\right)_{2} \mathrm{X}_{2}$ e haletos metálicos do tipo $\mathrm{MX}_{4}$ de platina e paládio ${ }^{19-20}$. Seus estudos mostraram excelentes resultados para o cálculo de geometrias e freqüências na região de 
infravermelho, com emprego de métodos RHF e DFT/B3LYP. Neste trabalho determinaram-se fatores de escalonamento variando entre 1,10 e 1,16, com as maiores bases exibindo os melhores resultados.

\section{CÁLCULOS}

Neste trabalho analisamos a capacidade de diversos modelos em reproduzir estruturas, geometrias, energias conformacionais e as respectivas freqüências vibracionais dos complexos em pauta. Empregamos vários níveis de cálculo e conjuntos de base. No tocante aos modelos de cálculo realizamos cálculos Hartree-Fock restritos, doravante RHF, teoria de perturbações Moller-Plessett em segunda ordem, denominada MP2 daqui por diante, além de cálculos baseados no funcional densidade empregando o funcional B3LYP, doravante denominado DFT.

Quanto aos conjuntos de base, empregamos bases simples, estendidas e também aquelas de uso combinado com pseudo-potenciais. A base e o pseudo-potencial de Stevens, Basch e Krauss ${ }^{21}$, foram empregados, quer representando exclusivamente o metal, daqui por diante SBK, quer representando todos os elementos pesados, i.e. metal e halogeneos, no caso dito SBK-X. A modificação de JansenCundari $^{22,23}$ ao pseudo-potencial anterior, SBKJC, também foi empregada. Também analisamos o comportamento do pseudo-potencial proposto por Hay e Wadt de Los Alamos ${ }^{24-26}$, doravante HW. Nos casos analisados SBK e SBK-X os demais elementos foram sempre representados por bases atômicas do tipo STO-3G ${ }^{27}$. Posteriormente à análise caso a caso, realizamos uma análise gráfica/estatística das freqüências calculadas quando confrontadas àquelas experimentalmente obtidas, abordagem que julgamos permitir uma discussão mais rica e significativa que a análise caso a caso das moléculas modelo.

Todos os cálculos foram realizados em um cluster Linux Red Hat 7.2 rodando em computadores pessoais Intel 350 e $930 \mathrm{MHz}$, fazendo uso do programa GAMESS ${ }^{28}$, versão 6 . Os critérios de convergência empregaram 0,0001 Hartree/Bohr de tolerância no gradiente. O método de cálculo empregou a aproximação quadrática.

\section{RESULTADOS}

\section{Complexos de Zinco}

\section{Diaminodiclorozinco(II) [ $\left.\mathrm{Zn}\left(\mathrm{NH}_{3}\right)_{2} \mathrm{Cl}_{2}\right]$}

O diaminodiclorozinco(II) foi estudado por diversos grupos experimentais, empregando espectroscopia IV/Raman com substituição isotópica de hidrogênio e nitrogênio ${ }^{11,29-30}$. A sua geometria, determinada por difração de raios-X, foi estudada por Nakamoto, Takemoto e $\mathrm{Chow}^{31}$. Todos os cálculos realizados com este complexo geraram geometrias tetraédricas do grupo de ponto $\mathrm{C}_{2 \mathrm{v}}$ Entretanto, dois confôrmeros são possíveis dependendo da posição dos grupos amônia entre si. Na primeira possibilidade, estrelada, o hidrogênio coplanar ao plano $\mathrm{N}-\mathrm{Zn}-\mathrm{N}$ situa-se externamente, enquanto na segunda o hidrogênio apresenta-se fechando o anel H-N-Zn-N-H, dito eclipsado. A geometria de menor energia é a estrelada, segundo Nakamoto e Takemoto ${ }^{31}$.

Por sua estrutura simples, com carga concentrada sobre o metal e grupos amino lábeis, os diversos cálculos mostraram excelente acordo com resultados experimentais. Obtiveram-se geometrias tetraédricas, cujos principais parâmetros geométricos são apresentados na Tabela 1. Nela pode-se observar que os resultados envolvendo pseudopotenciais são próximos aos experimentais ${ }^{29}$, com erros nas distâncias não superiores a 0,07 ̊̊ para a distância Zn-N e 0,15 ̊̊ para a distância $\mathrm{Zn}-\mathrm{Cl}$. Via de regra, o tratamento da energia de correlação mostrou distâncias de ligação metal-ligante menores que aquelas encontradas no modelo RHF, particularmente com as distâncias $\mathrm{Zn}-\mathrm{N}$ e $\mathrm{Zn}-\mathrm{Cl}$, cujos menores valores previstos foram determinados com o modelo MP2, enquanto o modelo DFT gerava resultados intermediários. Vê-se também cálculos com a base SBK-X gerando uma geometria de caráter mais "plano", exibindo maiores ângulos $\mathrm{N}-\mathrm{Zn}-\mathrm{N}$ e $\mathrm{Cl}-\mathrm{Zn}-\mathrm{Cl}$ que aqueles obtidos por outros cálculos. Curiosamente, o melhor resultado para distâncias de ligação foi obtido com o uso deste tipo de pseudopotencial. Para este complexo os resultados do pseudopotencial HW mostrou resultados similares aos exibidos pelas variações SBK e SBKJC.

Tabela 1. Principais parâmetros geométricos calculados para o complexo diamindiclorozinco(II) comparados a resultados experimentais $^{31}$ (distâncias em $\AA$ e ângulos em graus)

\begin{tabular}{lcccc}
\hline & $\mathrm{r}(\mathrm{Zn}-\mathrm{N})$ & $\mathrm{r}(\mathrm{Zn}-\mathrm{Cl})$ & $\theta(\mathrm{N}-\mathrm{Zn}-\mathrm{N})$ & $\theta(\mathrm{Cl}-\mathrm{Zn}-\mathrm{Cl})$ \\
\hline RHF & & & & \\
SBK & 2,08 & 2,13 & 103,2 & 126,6 \\
SBK-X & 2,03 & 2,31 & 119,5 & 131,9 \\
SBKJC & 2,09 & 2,13 & 101,8 & 125,8 \\
HW & 2,07 & 2,16 & 102,9 & 125,3 \\
MP2 & & & & \\
SBK & 2,02 & 2,12 & 104,9 & 123,8 \\
SBK-X & 1,98 & 2,29 & 121,5 & 128,5 \\
SBKJC & 2,03 & 2,11 & 103,6 & 122,8 \\
HW & 2,04 & 2,16 & 103,9 & 122,9 \\
DFT & & & & \\
SBK & 2,04 & 2,14 & 109,6 & 123,8 \\
SBK-X & 2,02 & 2,30 & 117,2 & 131,2 \\
SBKJC & 2,06 & 2,14 & 108,6 & 122,5 \\
HW & 2,07 & 2,19 & 108,7 & 122,6 \\
\hline Exp. & 2,02 & 2,27 & & \\
\hline
\end{tabular}

Analisando a barreira conformacional em torno da ligação de amônia, todos os cálculos mostraram a conformação estrelada como a mais estável, em concordância com os resultados de Nakamoto e Takemoto $^{29}$, entretanto a barreira de energia conformacional mostrou-se muito pequena, da ordem de $0,2 \mathrm{~kJ} / \mathrm{mol}$ no cálculo RHFHW, o que torna difícil o cálculo de freqüências de torção ao redor desta ligação. Creditamos a este pequeno valor o surgimento de frequiências imaginárias envolvendo o modo torção da amônia. Nem o cálculo acurado da geometria de energia mínima, uso de diferentes formas de cálculo da matriz hessiana, analítico quando possível e numérico com um ou dois pontos de cálculo, ou ainda a pesquisa no deslocamento utilizado, eliminou as freqüências imaginárias encontradas. Os valores encontrados dependem da base e do tipo de cálculo, mas situam-se em torno de $10 \mathrm{~cm}^{-1}$. Assim sendo, fomos obrigados a considerar, daqui por diante, a discussão dos modos de vibração torsionais como rotações internas livres, e serão omitidos de nossa análise neste trabalho.

$\mathrm{Na}$ análise das freqüências, observa-se uma sistemática superestimativa das frequiências de vibração, tal como pode ser observado na Tabela 2. Adicionalmente vê-se que o cálculo teórico mostra grande precisão na estimativa de frequiências envolvendo estiramentos $\mathrm{NH}$, pois neles o desvio padrão encontrado é da ordem de $11,6 \mathrm{~cm}^{-1}$, enquanto que nos estiramentos de ligações metálicas, este erro é sete vezes superior. O resultado que mais chama a atenção é do estiramento $\mathrm{Zn}-\mathrm{Cl}$ calculado com o modelo RHF/SBK, que superestima por um fator de dois a banda experimental. Claramente não é possível obter o mesmo nível de acuracidade no trabalho com metais, que naquele envolvendo a previsão de freqüências vibracionais de compostos orgânicos. Os resultados envolvendo outras bases 
mostram um comportamento claro, no qual há uma progressiva diminuição nas freqüências calculadas na ordem RHF $>$ MP2 $>$ DFT entre as freqüências de estiramento e deformação angular da amônia, isto é, modos que não envolvem o metal. Nos modos de "rocking" a ordem está invertida entre os modelos MP2 e DFT, (RHF > DFT > MP2). Via de regra, nos modos envolvendo o metal, não foi possível encontrar um comportamento bem definido.

Tabela 2. Frequiências representativas calculadas $\left(\mathrm{cm}^{-1}\right)$ para o complexo diamindiclorozinco (II) comparadas a resultados experimentais $^{11}$

\begin{tabular}{lccccc}
\hline & $v_{\mathrm{s}}(\mathrm{NH})$ & $\delta_{\mathrm{s}}(\mathrm{HNH})$ & $\rho\left(\mathrm{NH}_{3}\right)$ & $v_{\mathrm{as}}(\mathrm{ZnN})$ & $v_{\mathrm{as}}(\mathrm{ZnCl})$ \\
\hline RHF & & & & & \\
SBK & 3884 & 1408 & 701 & 400 & 502 \\
SBK-X & 3875 & 1465 & 767 & 471 & 337 \\
SBKJC & 3880 & 1387 & 693 & 395 & 501 \\
HW & 3884 & 1470 & 738 & 685 & 466 \\
MP2 & & & & & \\
SBK & 3618 & 1344 & 691 & 440 & 504 \\
SBK-X & 3611 & 1385 & 753 & 507 & 339 \\
SBKJC & 3612 & 1324 & 684 & 435 & 502 \\
HW & 3612 & 1413 & 716 & 450 & 457 \\
DFT & & & & & \\
SBK & 3533 & 1309 & 697 & 404 & 476 \\
SBK-X & 3536 & 1312 & 723 & 440 & 333 \\
SBKJC & 3537 & 1272 & 691 & 396 & 466 \\
HW & 3528 & 1369 & 719 & 431 & 428 \\
\hline Exp. & 3258 & 1268 & 668 & 422 & 285 \\
\hline
\end{tabular}

\section{Diamindibromozinco(II) $\left[\mathrm{Zn}\left(\mathrm{NH}_{3}\right)_{2} \mathrm{Br}_{2}\right]$}

O diamindibromozinco(II) é um composto tetraédrico de simetria $\mathrm{C}_{2 \mathrm{v}}$. A substituição do cloro pelo bromo aumenta a distância de ligação metal-halogêneo de 2,27 ̊̊ para 2,38 ̊. Em sistemas químicos similares, a substituição de elementos ou grupos por outros menos eletronegativos leva ao enfraquecimento de todas as constantes de força. Isto é observado na diminuição das freqüências de estiramento Zn-N de $258 \mathrm{~cm}^{-1}$ no diamindiclorozinco(II), para $212 \mathrm{~cm}^{-1}$ no diamindibromozinco(II). Deve-se observar o comportamento da base RHF/SBK, que exibe uma relativa insensibilidade em relação à troca dos halogenios no que diz respeito a distâncias de ligação, onde se obteve resultados de 2,02, 2,03 e 2,04 $\AA$ nos complexos de zinco com $\mathrm{Cl}, \mathrm{Br}$ e I. É também visível que esta base gera os menores resultados para as distâncias de ligação metal-halogenio, fato que, consistentemente, gerará as maiores frequiências calculadas. À luz desta experiência, desaconselhamos o uso de pseudopotenciais centrados exclusivamente no metal.

No tocante às freqüências, Tabela 3 , constata-se o mesmo padrão de resultados, com estiramentos sistematicamente superestimados na ordem RHF > MP2 > DFT, enquanto que nas deformações angulares ocorre um comportamento errático. É particularmente notável o fato que as frequiências calculadas com ambos os átomos descritos por pseudopotencias, SBK-X, mostram os melhores resultados, enquanto para aquelas envolvendo átomos diversos o resultado é um dos piores.

\section{Diamindiiodozinco(II) [ $\left.\mathrm{Zn}\left(\mathrm{NH}_{3}\right)_{2} I_{2}\right]$}

A substituição do bromo pelo iodo acentua as diferenças já relatadas no caso anterior, aumentando a distância metal-halogeneo. A distância metal-nitrogênio pouco se altera, em face de seu acentuado caráter covalente. Os resultados obtidos são, em grande medida, similares àqueles já mencionados para o caso anterior, com a base
Tabela 3. Freqüências $\left(\mathrm{cm}^{-1}\right)$ representativas calculadas para o diamindibromozinco(II) comparadas a resultados experimentais ${ }^{12}$

\begin{tabular}{lccccc}
\hline & $v_{\mathrm{s}}(\mathrm{NH})$ & $\delta_{\mathrm{s}}(\mathrm{HNH})$ & $\rho(\mathrm{NH} 3)$ & $v_{\text {as }}(\mathrm{ZnN})$ & $v_{\text {as }}(\mathrm{ZnBr})$ \\
\hline RHF & & & & & \\
SBK & 3882 & 1396 & 687 & 377 & 398 \\
SBK-X & 3868 & 1468 & 764 & 469 & 251 \\
SBKJC & 3879 & 1403 & 711 & 410 & 368 \\
HW & 3884 & 1479 & 747 & 428 & 361 \\
MP2 & & & & & \\
SBK & 3610 & 1336 & 679 & 416 & 412 \\
SBK-X & 3601 & 1385 & 746 & 503 & 253 \\
SBKJC & 3612 & 1332 & 700 & 447 & 371 \\
HW & 3612 & 1420 & 725 & 453 & 356 \\
DFT & & & & & \\
SBK & 3519 & 1295 & 672 & 377 & 397 \\
SBK-X & 3529 & 1317 & 724 & 438 & 248 \\
SBKJC & 3527 & 1287 & 706 & 405 & 348 \\
HW & 3528 & 1377 & 727 & 431 & 334 \\
\hline Exp. & 3247 & 1244 & 678 & 415 & 212 \\
\hline
\end{tabular}

SBK-X exibindo distâncias de ligação em bom acordo com resultados experimentais e ângulos centrais ( $\mathrm{N}-\mathrm{Zn}-\mathrm{N}$ e X-Zn-X) um pouco maiores. As tendências, já descritas anteriormente, cumprem-se também no caso do diamindiiodozinco(II), com freqüências superestimadas na ordem RHF $>$ MP2 > DFT. Desta forma, não teceremos quaisquer considerações especiais, legando ao leitor as Tabelas 4 e 5 onde são apresentadas as geometrias e frequiências vibracionais apuradas.

Tabela 4. Parâmetros geométricos para o complexo de diamindiiodozinco(II) comparados a resultados experimentais ${ }^{32}$ (distâncias em $\AA$ e ângulos em graus)

\begin{tabular}{lcccc}
\hline & $\mathrm{r}(\mathrm{Zn}-\mathrm{N})$ & $\mathrm{r}(\mathrm{Zn}-\mathrm{I})$ & $\theta(\mathrm{N}-\mathrm{Zn}-\mathrm{N})$ & $\theta(\mathrm{I}-\mathrm{Zn}-\mathrm{I})$ \\
\hline RHF & & & & \\
SBK & 2,10 & 2,34 & 95,7 & 127 \\
SBK-X & 2,03 & 2,65 & 115,4 & 129,9 \\
SBKJC & 2,07 & 2,50 & 104,6 & 125,8 \\
HW & 2,06 & 2,51 & 104,6 & 125,7 \\
MP2 & & & & \\
SBK & 2,04 & 2,29 & 96,6 & 126,3 \\
SBK-X & 1,98 & 2,60 & 116,3 & 127,1 \\
SBKJC & 2,02 & 2,46 & 105,8 & 124,2 \\
HW & 2,04 & 2,50 & 105,6 & 124,6 \\
DFT & & & & \\
SBK & 2,08 & 2,29 & 98,7 & 126,9 \\
SBK-X & 2,02 & 2,62 & 114,6 & 129,5 \\
SBKJC & 2,05 & 2,49 & 110,1 & 124,7 \\
HW & 2,07 & 2,54 & 109,7 & 124,7 \\
\hline Exp. & 2,10 & 2,53 & & \\
\hline
\end{tabular}

\section{Complexos de Cádmio}

Diamindiclorocádmio(II) [Cd( $\left.\left.\mathrm{NH}_{3}\right)_{2} \mathrm{Cl}_{2}\right]$

O diamindiclorocádmio(II) é um complexo planar, pertencendo ao grupo de ponto $\mathrm{C}_{2 \mathrm{v}}$, no qual os ângulos ao redor do cádmio valem $90^{\circ}$ e os átomos de cloro e nitrogênio situam-se em um mesmo plano. Este complexo foi estudado primeiramente por Barrow, Krueger 
Tabela 5. Freqüências $\left(\mathrm{cm}^{-1}\right)$ calculadas para o complexo de diamindiiodozinco(II) comparadas a resultados experimentais ${ }^{13}$

\begin{tabular}{lccccc}
\hline & $v_{\mathrm{s}}(\mathrm{NH})$ & $\delta_{\mathrm{s}}(\mathrm{HNH})$ & $\rho\left(\mathrm{NH}_{3}\right)$ & $v_{\text {as }}(\mathrm{ZnN})$ & $v_{\text {as }}(\mathrm{ZnI})$ \\
\hline RHF & & & & & \\
SBK & 3880 & 1404 & 698 & 370 & 295 \\
SBK-X & 3864 & 1472 & 765 & 464 & 206 \\
SBKJC & 3876 & 1418 & 730 & 422 & 287 \\
HW & 3884 & 1493 & 762 & 436 & 288 \\
MP2 & & & & & \\
SBK & 3604 & 1347 & 688 & 406 & 320 \\
SBK-X & 3594 & 1389 & 743 & 493 & 214 \\
SBKJC & 3608 & 1351 & 714 & 455 & 293 \\
HW & 3614 & 1433 & 736 & 457 & 287 \\
DFT & & & & & \\
SBK & 3513 & 1268 & 649 & 358 & 307 \\
SBK-X & 3524 & 1335 & 725 & 434 & 206 \\
SBKJC & 3536 & 1307 & 720 & 412 & 279 \\
HW & 3531 & 1392 & 736 & 434 & 272 \\
\hline Exp. & 3236 & 1242 & 676 & 406 & 177 \\
\hline
\end{tabular}

e Basolo ${ }^{33}$, que promoveram a análise do seu espectro de infravermelho. Posteriormente, Clark e Williams ${ }^{30}$ realizaram a atribuição dos modos vibracionais de esqueleto e realizaram cálculos aproximados para as constantes de força. Na década de 90, Tellez e colaboradores ${ }^{14,15}$ promoveram estudos com os seus isótopos deuterados e de ${ }^{15} \mathrm{~N}$.

Nos cálculos, a substituição do zinco pelo cádmio mostrou uma pequena queda na qualidade dos resultados, e nenhum cálculo foi capaz de reproduzir a geometria planar dos experimentos de MacGillavry ${ }^{34}$. Todas as geometrias geradas possuem a forma levemente tetraédrica ao redor do cádmio. No tocante a distâncias de ligação, o pseudopotencial combinado SBK-X foi aquele que mostrou os melhores resultados, especialmente quando empregado em cálculos DFT.

Um aspecto interessante é o de que, embora as distâncias de ligação experimentais zinco-nitrogênio pouco se alterem com a mudança do halogenio, nos cálculos dos complexos de cádmio estes se mostraram sensíveis a essa alteração com o aumento dos comprimentos de ligação $\mathrm{Cd}-\mathrm{N}$. As distâncias de ligação $\mathrm{Cd}-\mathrm{Cl}$, em todos os pseudopotenciais, ficaram abaixo dos resultados experimentais e todas em torno de $2,3 \AA$, exceto o pseudopotencial SBK-X que confirmou seu melhor comportamento com resultados na faixa de $2,5 \AA$ frente ao resultado experimental de $2,71 \AA$. Em face do resultado adverso para a geometria quase-planar, nos eximiremos de apresentar as tabelas associadas.

A variação dos comprimentos de ligação tem influência direta nos respectivos modos de vibração, i.e., quando um particular cálculo gera distâncias de ligação $\mathrm{Cd}-\mathrm{Cl}$ menores que a experimental, as respectivas bandas de estiramento são superestimadas. Também isto ocorre para o estiramento assimétrico $\mathrm{Cd}-\mathrm{N}$, cujos resultados para a distância de ligação são, em geral, maiores que o esperado para todas as bases. Consistentes são os resultados para o pseudopotencial SBK-X, que gera a menor distância de ligação Cd-N e a maior freqüência de vibração associada. As bandas de média energia, tal como o "rocking" da amônia, apresentam resultados que variam pouco com o método de cálculo.

Deve-se observar que as frequiências de estiramento estão todas superestimadas na ordem RHF $>$ MP2 $>$ DFT, com o último apresentando um erro de apenas $8 \%$ para o estiramento simétrico $v_{\mathrm{s}}(\mathrm{NH})$ no pseudopotencial HW.
Diamindibromocádmio (II) $\left[\mathrm{Cd}\left(\mathrm{NH}_{3}\right)_{2} \mathrm{Br}_{2}\right]$

O complexo diamindibromocádmio(II) teve o seu espectro de IV atribuído por Clark e Williams ${ }^{30}$ em um trabalho inicial. Posteriormente foi estudado por Téllez e colaboradores ${ }^{16}$, que realizaram um cuidadoso trabalho nas atribuições de bandas de IV e de espalhamento Raman com base nos experimentos de atribuição isotópica realizados.

Os parâmetros geométricos presentes na literatura ${ }^{34}$ apontam para uma estrutura similar à do complexo análogo de cloro, na qual a maior diferença é aumento no comprimento da ligação $\mathrm{Cd}-\mathrm{X}$, de 2,71 Å no complexo de cloro a 2,86 ̊̊ no composto análogo de bromo. Os cálculos mostraram comportamento semelhante a aqueles obtidos no caso do complexo anterior. Com o pseudopotencial SBK/RHF os resultados convergiram para uma estrutura planar em conformidade com os relatados na literatura. Todavia os comprimentos de ligação $\mathrm{Cd}-\mathrm{N}$ e $\mathrm{Cd}-\mathrm{Br}$ mostraram grande diferença para com os resultados experimentais, com a distância $\mathrm{Cd}-\mathrm{N}$ sistematicamente maior, enquanto a distância $\mathrm{Cd}-\mathrm{Br}$ sempre subestimada. Não exibiremos a tabela correspondente, porém sabe-se que os melhores resultados obtidos para a geometria são aqueles do pseudopotencial SBK-X, mostrando uma estrutura de ângulos menores que $180^{\circ}$, porém com comprimentos de ligação coerentes com os dados experimentais.

As frequiências vibracionais exibem comportamento já discutido, com as bandas de mais alta energia superestimadas na forma RHF $>$ MP2 > DFT. A base SBK mostrou-se especialmente problemática no modelo RHF, as distâncias exageradas para as ligações $\mathrm{Cd}-\mathrm{N}$ e Cd-Br. Consistentemente este pseudopotencial mostrou os piores resultados nas respectivas frequiências de estiramento. O ECP combinado a SBK-X mostrou comportamento similar ao de outros complexos, i.e., quando o estiramento envolve átomos que possuem o ECP, obtém-se excelentes resultados, enquanto no caso contrário, este apresenta os piores resultados.

\section{Diamindiiodocádmio(II) [Cd( $\left.\left.\mathrm{NH}_{3}\right)_{2} \mathrm{I}_{2}\right]$}

Este composto teve o seu espectro de IV e Raman estudado por Téllez e colaboradores ${ }^{17}$. Sua geometria é análoga a dos complexos anteriores, com mínimas diferenças no comprimento da ligação metal-halogêneo ${ }^{35,36}$. Os cálculos revelaram uma geometria planar, em nível RHF e MP2. No entanto os mesmos problemas, com respeito as ligações $\mathrm{Cd}-\mathrm{N}$ e $\mathrm{Cd}-\mathrm{Br}$, se manifestaram nestes cálculos.

No tocante a frequiências vibracionais observa-se comportamento análogo ao de outros complexos de cádmio.

\section{Complexo de mercúrio}

Íon diamin-mercúrio (II) $\left[\mathrm{Hg}\left(\mathrm{NH}_{3}\right)_{2}\right]^{2+}$

$\mathrm{O}$ íon complexo diamin-mercúrio (II) é linear ${ }^{35}$, pertencendo ao grupo de ponto $\mathrm{D}_{3 \mathrm{~d}}$. $\mathrm{O}$ seu espectro de IV e Raman foi estudado por Téllez e Díaz ${ }^{18}$, com o objetivo de eliminar dúvidas a respeito das frequiências de vibração de esqueleto deste complexo e, para tanto, fez uso dos espectros de IV e Raman dos isotopômeros, deuterados e de ${ }^{15} \mathrm{~N}$, deste composto.

Realizamos cálculos empregando esta estrutura e simetria $D_{3 \mathrm{~d}}$. Alguns cálculos não puderam ser realizados pois a base HW não possui parametrização para este pseudopotencial no mercúrio. Os comprimentos de ligação $\mathrm{Hg}-\mathrm{N}$ apresentam grande reprodutibilidade nos diferentes níveis de cálculo e concordância excelente com resultados da literatura ${ }^{37}$. No que confere às frequiências de vibração, excelentes resultados foram obtidos, como pode ser visto na Tabela 6 .

\section{ANÁLISE GRÁFICA}

De modo a empreender uma análise mais completa do compor- 
Tabela 6. Freqüências calculadas $\left(\mathrm{cm}^{-1}\right)$ para o íon diaminmercúrio(II) comparados a resultados experimentais ${ }^{18}$

\begin{tabular}{lcccc}
\hline & $v_{\mathrm{s}}(\mathrm{NH})$ & $\delta_{\mathrm{s}}(\mathrm{HNH})$ & $\rho(\mathrm{NH} 3)$ & $v_{\text {as }}(\mathrm{HgN})$ \\
\hline RHF & & & & \\
SBKJC & 3787 & 1618 & 960 & 565 \\
SBKJC & 3782 & 1607 & 956 & 560 \\
MP2 & & & & \\
SBK & 3495 & 1506 & 942 & 570 \\
SBKJC & 3488 & 1493 & 936 & 564 \\
DFT & & & & \\
SBK & 3441 & 1476 & 902 & 539 \\
SBKJC & 3439 & 1459 & 893 & 532 \\
\hline Exp. & 3210 & 1275 & 720 & 518 \\
\hline
\end{tabular}

tamento de cada modelo e do uso de pseudopotencias na previsão de freqüências vibracionais de complexos inorgânicos, decidimos lançar todas as freqüências vibracionais conhecidas contra as calculadas, em gráficos por métodos e pseudopotencias. Os resultados exibirão um comportamento de família que pode ser útil na observação de regularidades de difícil identificação, além de permitir correções que reduzam o erro total apurado. Ramos e Hollauer ${ }^{10}$ construíram um modelo estatístico baseado no modelo AM1 que, sistematicamente, reduziu à metade o erro médio de cálculo.

Lançamos todos os resultados experimentais e calculados conhecidos em gráficos, e ajustamos a melhor reta por critério de mínimos erros quadráticos médios abaixo descrita:

I) $f_{\text {calc }}=a f_{\text {exp }}+b$

Para esta análise determinamos o desvio quadrático acumulado (SD), e o coeficiente de correlação (R). Como o número de modos considerados em cada modelo e pseudo-potencial são os mesmos, à exceção da base SBK-X que não contabiliza o complexo de mercúrio, pode-se orientar o estudo que realizamos a partir dos valores do desvio quadrático acumulado em cada modelo.

As Figuras de 1 a 4 exibem a curva de freqüências calculadas contra as experimentais, acompanhada dos respectivos desvios padrões e coeficiente de correlação, para o cálculo MP2 empregando

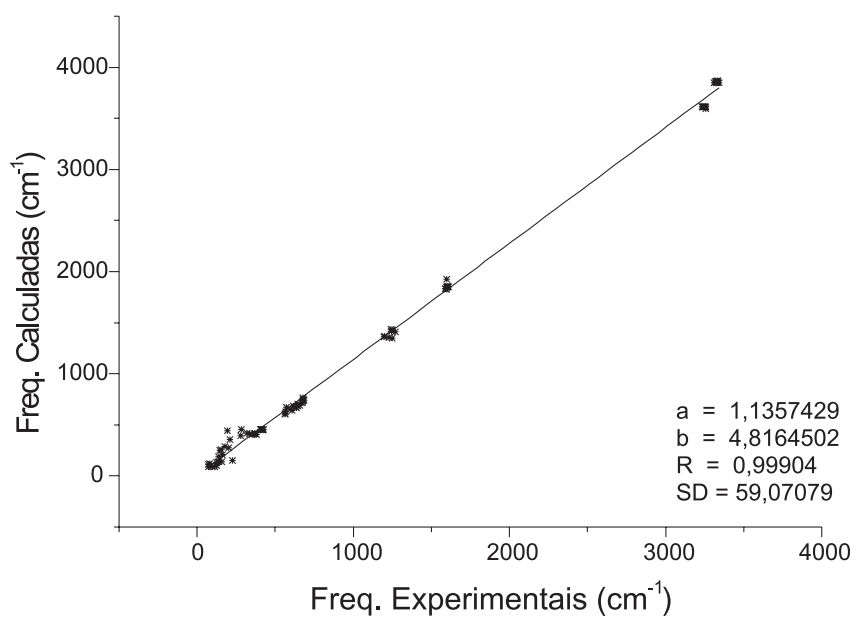

Figura 1. Gráfico das freqüências calculadas versus as freqüências experimentais para toda a família de complexos usando o modelo MP2. O pseudopotencial empregado foi o $\mathrm{HW}$

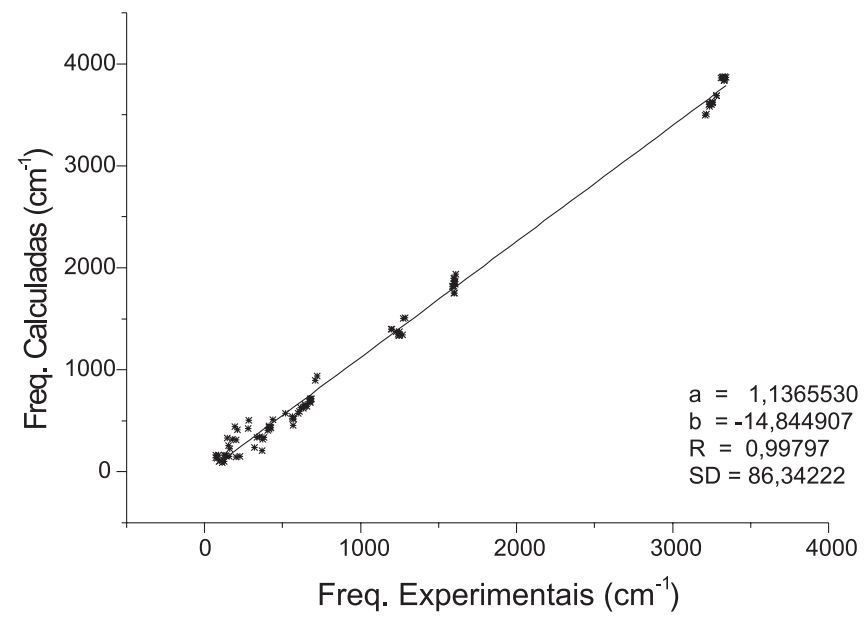

Figura 2. Gráfico das freqüências calculadas versus as freqüências experimentais para toda a família de complexos usando o modelo MP2. O pseudopotencial empregado foi o SBK

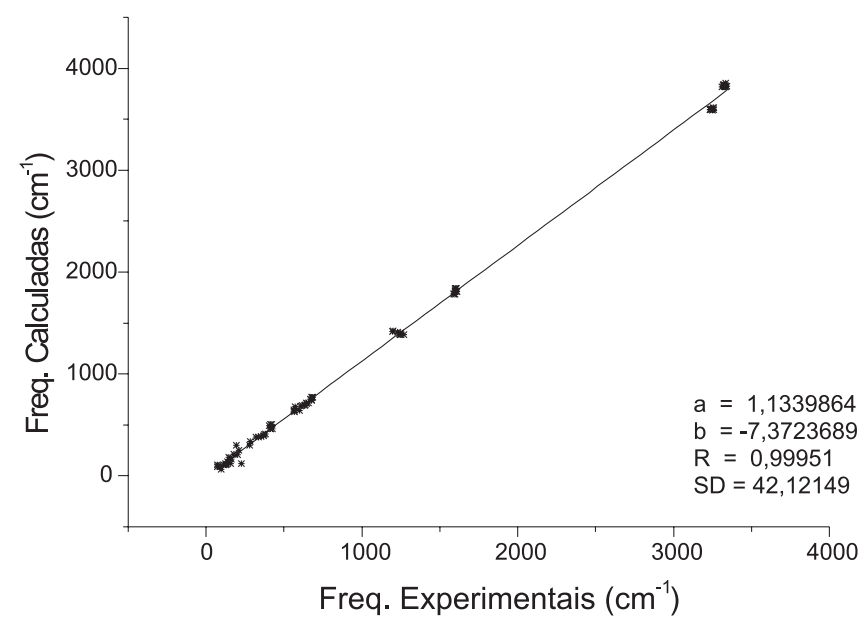

Figura 3. Gráfico das freqüências calculadas versus as freqüências experimentais para toda a família de complexos usando o modelo MP2. O pseudopotencial empregado foi o SBK-X

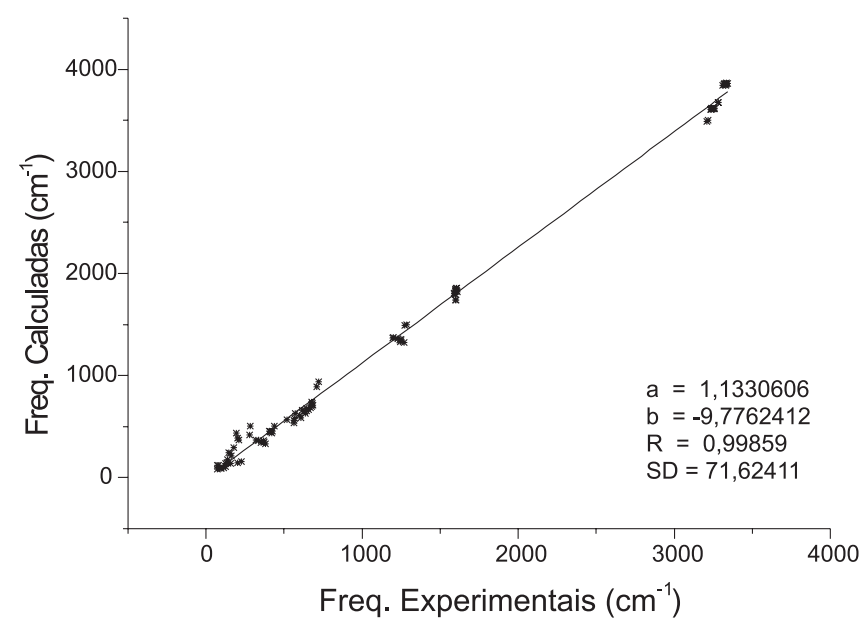

Figura 4. Gráfico das freqüencias calculadas versus as freqüências experimentais para toda a família de complexos usando o modelo MP2. O pseudopotencial empregado foi o SBKJC 
cada um dos pseudopotencias utilizados neste trabalho. A análise destas curvas mostra que, na região de 3400-3600 $\mathrm{cm}^{-1}$, os estiramentos $\mathrm{NH}$ exibem um coeficiente angular mais elevado que aquele observado em baixa frequiência.

A Figura 5, ilustra duas curvas de ajuste, diferenciando as regiões de baixa e alta freqüência. Os desvios padrões apurados são, neste caso, muito favoráveis ao ajuste duplo.

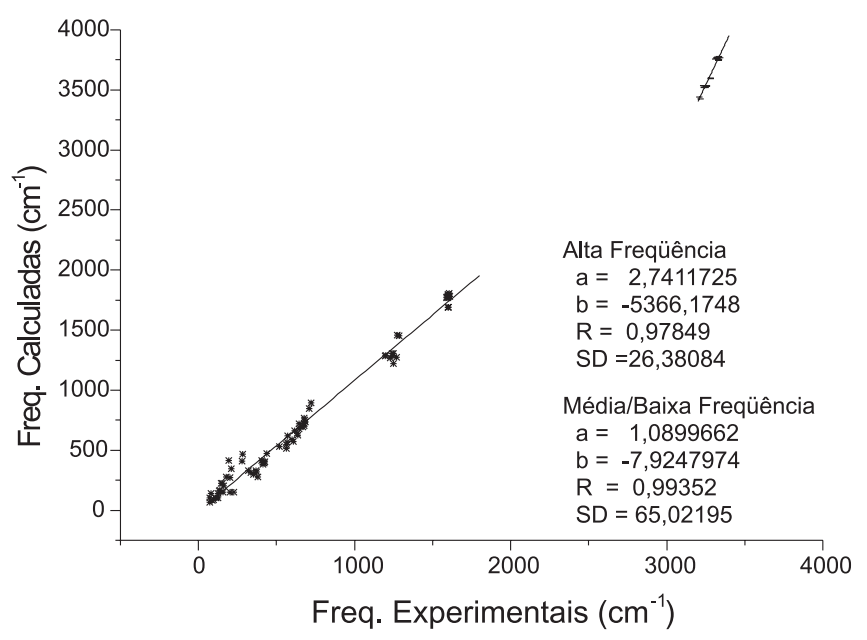

Figura 5. Gráfico das freqüências calculadas versus as freqüências experimentais para toda a família de complexos usando o modelo DFT/ SBKJC. Nesta figura, uma curva descrevendo "twistings", deformações angulares e estiramentos de baixa energia, foi obtida no canto inferior à esquerda do gráfico. No canto superior à direita, apresentamos um gráfico específico para os estiramentos $\mathrm{NH}$ acima de $3000 \mathrm{~cm}^{-1}$

A partir destes valores é possível concluir que o pseudopotencial SBK, utilizado para todos os elementos pesados, SBK-X, foi aquele de melhor resultado. O valor de SD acumulado situa-se em torno de 40, dez abaixo do modelo HW. O segundo melhor resultado surpreendeu aos autores, pois foi o pseudopotencial de Hay-Wadt. Este pseudopotencial, ao longo de nossas discussões, não mostrou qualquer resultado especialmente acurado. Apesar disto seus resultados são estatísticamente acurados, i.e., mostram um comportamento de superestimativa regular de todos os modos de vibração, fato que leva a um melhor comportamento estatístico, além de permitir um processo de correção que minimize estes erros. Apurou-se um valor situado na faixa de 53-63 para o SD. Os pseudopotenciais SBKJC e SBK, nesta ordem, terminam a seqüência estudada neste artigo. A Tabela 7 mostra os resultados obtidos para os coeficientes angulares, lineares, o erro quadrático acumulado e o coeficiente de correlação.

Uma particularidade interessante a observar diz respeito à clara observação da necessidade de modelos específicos ao tratamento das freqüências de estiramento $\mathrm{NH}$, situada na região de $3100-3200 \mathrm{~cm}^{-1}$. Para esta região, observa-se uma reta de coeficiente angular muito mais acentuado que a determinada pelo procedimento de mínimos quadrados. Considerando-se que as presentes freqüências foram identificadas visualmente, o que não permite grandes questionamentos, deve-se recomendar o uso de modelos específicos para estiramentos $\mathrm{NH}$

\section{CONCLUSÃO}

Analisamos quatro diferentes pseudo-potenciais, SBK-X, SBKJC, SBK e HW quando sujeitos a cálculos com três diferentes mode-
Tabela 7. Resultados para a análise gráfica das frequiências calculadas por método e conjunto de base. Apresenta-se o coeficiente angular, o coeficiente linear e o valor complementar ao coeficiente de correlação $\left(1-R^{2}\right)$

\begin{tabular}{lccc}
\hline & $\mathrm{A}$ & $\mathrm{B}\left(\mathrm{cm}^{-1}\right)$ & $\left(1-\mathrm{R}^{2}\right) \times 10^{3}$ \\
\hline RHF & & & \\
SBK & 1,236 & $-68,0$ & 4,3 \\
SBK-X & 1,228 & $-46,0$ & 0,8 \\
SBKJC & 1,228 & $-53,3$ & 2,7 \\
HW & 1,228 & $-34,3$ & 1,9 \\
MP2 & & & \\
SBK & 1,136 & $-14,8$ & 4,1 \\
SBK-X & 1,134 & $-7,8$ & 0,8 \\
SBKJC & 1,133 & $-9,8$ & 2,8 \\
HW & 1,136 & 4,8 & 1,9 \\
DFT & & & \\
SBK & 1,112 & $-24,6$ & 3,5 \\
SBK-X & 1,115 & $-24,3$ & 1,0 \\
SBKJC & 1,110 & $-20,1$ & 2,7 \\
HW & 1,108 & 1,4 & 1,7 \\
\hline
\end{tabular}

los tradicionalmente empregados no estudo de complexos metálicos, RHF, MP2 e DFT com relação à capacidade de previsão das freqüências de vibração de oito complexos metálicos amplamente estudados na literatura. Os resultados mostraram que o modelo SBK-X exibe os melhores resultados, específicos quando há a comparação direta entre geometrias e frequiências calculadas/experimentais e também no tocante a resultados estatísticos. Assim recomenda-se, quando se fizer uso do pseudopotencial SBK, utilizá-lo em todos os elementos vizinhos, da maneira mais estendida possível.

O segundo pseudopotencial recomendado é o proposto por Hay e Wadt, que exibiu bons resultados estatísticos. No tocante à superestimativa, os pseudopotencias alternam-se dependendo do modelo, mas no RHF o pseudopotencial que menos superestima as frequiências de vibração é o SBK-X, no modelo MP2 é o SBKJC (com o SBK-X logo após) e, finalmente, no modelo DFT com o pseudopotencial HW exibindo os melhores resultados.

Dentre os modelos confirmou-se aquilo que muitos trabalhos prévios já haviam relatado, que o modelo DFT é aquele de melhores resultados para frequiências de vibração, seguido do MP2 com resultados próximos. O modelo RHF é desaconselhável no caso de complexos metálicos.

Reforçando conclusões estabelecidas em trabalhos anteriores, a análise dos estiramentos $\mathrm{NH}$ na região de $3400-3600 \mathrm{~cm}^{-1}$ mostrou concordância muito melhor para um ajuste de duas retas, diferenciando as regiões de baixa e alta frequiência.

\section{AGRADECIMENTOS}

O presente trabalho não teria sido possível sem o apoio financeiro de diversas fontes, em caráter pessoal e institucional. Assim cumpre-nos agradecer bolsas de pesquisa conferidas ao estudante de iniciação científica, T. Giannerini, através do programa PIBIC/PROPP/ CNPq e as bolsas de pesquisador conferidas à C. A. T. Soto e E. Hollauer. Adicionalmente os recursos computacionais utilizados decorrem de projetos financiados por diversas agências e projetos, a saber CNPq(420026/98-3) e FAPERJ(E-26/150118/2001) na forma do prêmio Jovem Cientista. Por fim, agradecemos ao Programa de Pós-Graduação em Química da Universidade Federal Fluminense, pela contratação de C. A. T. Soto. 


\section{REFERÊNCIAS}

1. Zhou, X.; Mole, S. J.; Liu, R.; Vib. Spectrosc. 1996, 12, 73.

2. Hout Jr., R. F.; Levi, B. A.; Hehre, W. J.; J. Comput. Chem. 1982, 3, 234.

3. DeFrees, D. J.; McLean, A. D.; J. Chem. Phys. 1985, 82, 333.

4. Scott, A. P.; Random, L.; J. Phys. Chem. 1996, 100, 16502.

5. Hehre, W. J.; Radom, L.; Schleyer, P. V. R.; Pople, J. A.; Ab Initio Molecular Orbital Theory, John Wiley \& Sons: New York, 1986.

6. Dewar, M. J. S.; Ford, G. P.; J. Am. Chem. Soc. 1977, 99, 1685.

7. Healy, E. F.; Holder, A.; J. Mol. Struct. 1993, 281, 141.

8. Coolidge, M. B.; Marlin, J. E.; Stewart, J. J. P.; J. Comput. Chem. 1991, 12, 948 .

9. Fausto, R.; J. Mol. Struct. 1994, 323, 267.

10. Ramos, J. C. S.; Cardoso, S. P.; Hollauer, E.; Quim. Nova 1999, 22, 684.

11. Soto, C. A. T.; Cruspeire, L. D.; Can. J. Appl. Spectrosc. 1990, 35, 105.

12. Ishikawa, D. N.; Soto, C. A. T.; Vib. Spectrosc. 1994, 8, 87.

13. Soto, C. A. T.; Ishikawa, D. N.; Lara, J. G.; Spectrosc. Lett. 1998, $31,313$.

14. Soto, C. A. T.; de Souza, F. A.; Spectrosc. Lett. 1991, 24, 1209.

15. Ishikawa, D. N.; de Souza, F. A.; Soto, C. A. T.; Spectrosc. Lett. 1993, 26, 803.

16. Soto, C. A. T.; de Souza, F. A.; Spectrosc. Lett. 1993, 26, 793.

17. Soto, C. A. T.; Ishikawa, D. N.; Rev. Soc. Quim. Mex. 2000, 44, 139.

18. Soto, C. A. T.; Díaz, G.; J. Mol. Struct. 1981, 77, 213.

19. Zhang, L.; Wei, H.; Zhang, Y.; Guo, Z.; Zhu, L.; Spectrochim. Acta, Part A 2002, 58, 217 .
20. Zhang, L.; Zhang, Y.; Tao, H.; Sun, X.; Guo, Z.; Zhu, Z.; J. Mol. Struct. (Theochem.), no prelo.

21. Stevens, W. J.; Bash, H.; Krauss, M.; J. Chem. Phys. 1984, 81, 6026.

22. Stevens, W. J.; Krauss, M.; Bash, H.; Jasien, P. J.; Can. J. Chem. 1992 70,612 .

23. Cundari, T. R.; Stevens, W. J.; J. Chem. Phys. 1993, 98, 5555

24. Hay, P. J.; Wadt, W. R.; J. Chem. Phys. 1985, 82, 270.

25. Wadt, W. R.; Hay, P. J.; J. Chem. Phys. 1985, 82, 284

26. Hay, P. J.; Wadt, W. R.; J. Chem. Phys. 1985, 82, 299.

27. Hehre, W. J.; Stewart, R. F.; Pople, J. A.; J. Chem. Phys. 1969, 51, 2657.

28. Schmidt, M. W.; Balridge, K. K.; Boatz, J. A.; Elbert, S. T.; Gordon, M. S.; Jensen, J. J.; Koseki, S.; Matsunaga, N.; Nguyen, K. A.; Su, S.; Windus, T. L.; Dupuis, M.; Montgomery, J. A.; J. Comput. Chem. 1993, 14, 1347.

29. Perchard, C.; Novak, A.; Spectrochim. Acta, Part A 1970, 26, 871.

30. Clark, R. J. H.; Williams, C. S.; J. Chem. Soc. A 1966, 30, 1425.

31. Nakamoto, K.; Takemoto, J.; Chow, T. L.; Appl. Spectrosc. 1971, $25,352$.

32. Friess, R.; J. Am. Chem. Soc. 1930, 52, 3083.

33. Barrow, G. M.; Krueger, R. H.; Basolo, F.; J. Inorg. Nucl. Chem. 1956, 2, 340.

34. MacGillavry, C. H.; Bijvoet, J. M.; Z. Kristallogr. 1936, 94, 231.

35. MacGillavry, C. H.; Bijvoet, J. M.; J. M. Chem. Weekbl 1935, 32, 346.

36. MacGillavry, C. H.; Bijvoet, J. M.; Z. Kristallogr. 1973, 94, 821.

37. Nakagawa, I,; Shimanouchi, T.; Spectrochim. Acta 1966, 22, 759. 